\title{
An Agent-based Model to Study the Evolution of Service Systems through the Service Life Cycle
}

\author{
Chathura Rajapakse and Takao Terano \\ Department of Computational Intelligence and Systems Science, \\ Tokyo Institute of Technology, Japan \\ chathura@trn.dis.titech.ac.jp
}

\begin{abstract}
We introduce a unified concept of service life cycle (SLC) for the study of service system evolution. The need for this research arises from the fact that studying service ecosystems has become a critical need in the modern service economy. Adopting the view that a service ecosystem is a complex adaptive system of interacting service systems, we propose Agentbased Modeling methodology as a potential candidate for service research. Our agent-based model simulates an artificial market, in which one hypothetical service is being offered and customer agents interact with service provider agents to co-create value. The design of the model takes inputs from the service dominant logic, Kauffman's NKCS model and the ISPAR model of service system interactions. The simulation results reveal that the evolution of service providers follows a life cycle pattern, which we call the Service Life Cycle. We propose the SLC to be used as a reference to evaluate the performance of service providers in a service ecosystem, initiating a novel S-D logic based discussion on sustainability of business firms.
\end{abstract}

Keywords: Service Ecosystems, Service Science, Life cycle, Service Life cycle, ISPAR Model, NKCS Model

\section{Introduction}

The world is becoming characterized by services and we can observe a rapid growth in the percentage contribution of the services sector to the GDP of many countries in the world [1, 2]. According to Figure 1, this percentage is more than $70 \%$ in many of the world's most advanced economies and even many developing countries have a figure more than $50 \%$ [3]. However, the business logic of the market is still largely focusing on manufacturing and selling goods and based on this traditional Goods-Dominant logic, a service is the intangible equivalent of goods [4]. Unarguably, acting with yesterday's logic is not going to help businesses become competitive in today's (and future) market. This has given rise to an evergrowing array of questions that have significant implications for the success of firms, the well-being of societies and the quality of consumers' lives worldwide [1]. Consequently, understanding service systems to foster service innovation has become a critical need in this modern service economy [5]. In response, there emerges a new interdisciplinary research discipline called 'Service Science', which focuses it's inquiry on fundamental science, models, theories, and applications to drive service innovation, competition, and well-being through co-creation of value [1].

Contemporary service research has been largely influenced and benefited by the ServiceDominant Logic (S-D Logic). In fact, it has been most commonly cited as a catalyst theory in 
the future service research [1]. S-D logic brings in the systems thinking approach to the study of market interactions [6]. Based on eight key aspects, S-D logic proposes a fundamental shift in the mindset of looking at market interactions from traditional Goods-Dominant Logic [4]. In the view of Service-Dominant logic, a market is a network of resource integrating economic actors [6]. This integration of resources enables actors to apply their resources for the benefit of another actor in the network in the form of a service.

S-D logic rejects the traditional distinction between goods and services (i.e., service as the intangible equivalent of goods) but rather considers the relationship between them [4]. In other words, in the exchange of services between actors of a resource network, goods serve as means of integrating resources to deliver services. In the notion of S-D logic, value of a service cannot be added beforehand and exchanged for something (Ex. Money). Instead, the usefulness of resources from one source, i.e., value, has to be uniquely and phenomenologically determined by the beneficiary [6]. This process is called co-creation of value. Each instance of resource integration, service provision and value (co) creation, changes the nature of the network to some degree and thus the context for the next iteration, making the network not just a network but also a dynamic service ecosystem [6].

Actors of a service ecosystem could be anything such as individuals, organizations, schools, countries, animals or even worlds and planets, depending on the level of abstraction [6]. These actors of a service ecosystem can be generalized into an abstract concept called 'service systems' [6,7]. According to [8], service science combines organization and human understanding with business and technological understanding to categorize and explain the many types of service systems that exists as well as how service systems interact and evolve to co-create value. In other words, the core research issues of service science regarding the success of firms, the well-being of societies and the quality of consumers' lives worldwide could be addressed in the light of the evolution of service systems.

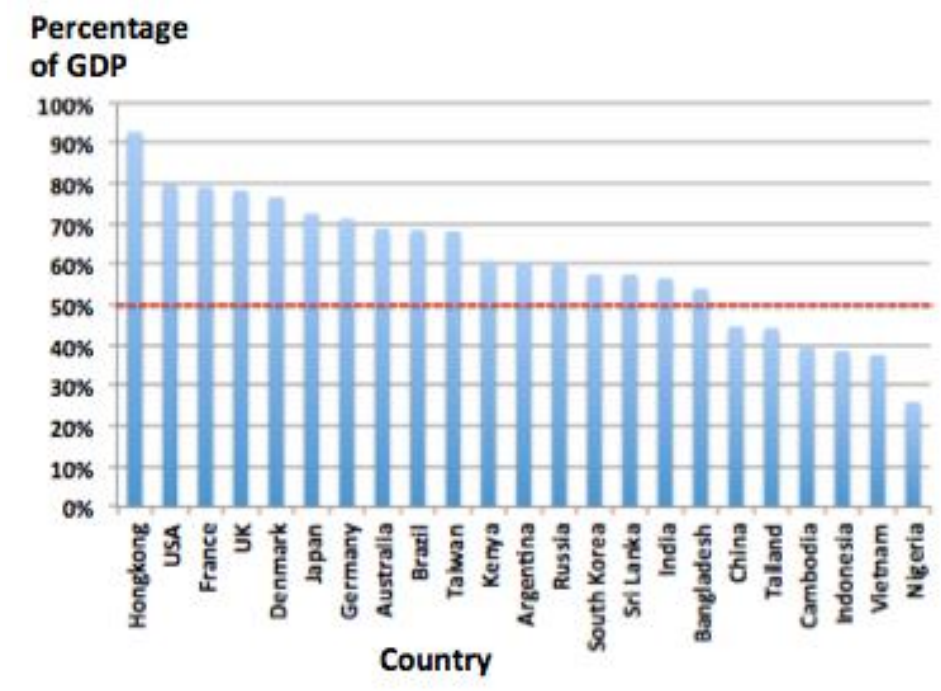

Figure 1. Contribution of service sector to the GDP by country

One particular way of depicting evolution is by means of a life cycle. Life cycle concept has been commonly used in traditional business management literature to understand evolution of certain phenomena and to propose respective strategies at different stages of evolution. One good example is the famous Product Life Cycle (PLC), which depicts the evolution of a product [9]. Similarly, in the domain of tourism, tourism area life cycle 
(TALC) has been proposed to understand the evolution of a tourist destination [10]. Both of these life cycles measure the frequency of consumption over time and enable to identify different phases of evolution such as introduction, growth, stagnation, decline, etc. to determine probable strategies for each phase for business performance. This same concept could be used in the domain of service ecosystems to study the evolution of service systems.

However, this study of the evolution of service systems is a challenging task. According to S-D logic, a service ecosystem comprising multiplicities of interacting service systems is a complex adaptive system [6]. As we could observe, current research in the service research frontiers is very often depending on the data collected through questionnaires and interviews. One particular challenge with the study of complex adaptive systems is the inability to analyze by decomposition due to the property of 'emergence' associated with complex adaptive systems [11]. The evolutionary pattern of service systems in a service ecosystem could be identified as an emerging pattern due to their interactions with other service systems. Therefore, research based on data collected through interviews and questionnaires is not adequate to get a holistic picture about the evolution of service systems. Moreover, an interdisciplinary research approach has been recommended for the service science research to develop necessary knowledge and tools [5].

In this research, we intend to study the evolution of service systems using the Agent-based Modeling (ABM) approach [12]. Due to its widespread use in the domain of complex adaptive systems, ABM has a potential as a research methodology in the domain of service science. Agent-based modeling consist of a number of interacting autonomous agents who are represented as computerized independent entities capable of acting locally in response to stimuli or to communication from other agents $[12,13,14]$. These local actions of each individual of a population of agents emerge various global complex patterns. In other words, these agents act as parts of a complex system, of which the macro-level properties can only be studied by letting the parts to interact with each other. Due to this reason, agent-based modeling has become a prominent technology in studying complex adaptive systems $[12,13]$. On the other hand, G-D logic is well established in the world and it makes real experiments to test an alternative dominant logic risky and difficult. Therefore, simulation approach could be recommended and agent-based modeling becomes an obvious solution.

This paper elaborates the details of an agent-based model of a service ecosystem developed with the objective of studying the evolution of service systems by means of analyzing a life cycle. The service ecosystem model is an artificial market model, which contains two types of service systems - Service Providers and Customers. The artificial market contains multiplicities of service systems (agents) of both types. All service providers offer one particular hypothetical service that satisfies a need of customers. We develop the model based on the fundamentals of S-D logic using two existing theoretical models as major building blocks. One is Kauffman's NKCS model [15] used to formalize the service ecosystem as a computational model of service systems. The other is the ISPAR model of service system interactions used to model the interactions between service systems [7].

In the analysis based on the initial results of the model, we basically focus on the evolution of service providers measuring the number of service interactions take place with each service provider at each time step over a longer period of time. Through this, we observe a life cycle pattern of evolution, which is similar to the known life cycle patterns such as the product life cycle and tourism area life cycle. We propose this new life cycle to be called Service Life Cycle (SLC) and argue that other existing life cycles could be brought under this common umbrella to initiate a novel S-D logic based 
discussion on the sustainability of business entities [16]. The rest of the paper is organized as below. Chapter two revisits the important literature in both service science and agent-based modeling. Chapter three explains our agent-based model in detail and chapter four presents the initial results. Chapter five contains a discussion based on results and chapter six provides concluding remarks.

\section{Literature Review}

According to [17], as a common standard, articles on agent-based modeling should include references to the theoretical background of the social or economic phenomenon that is investigated. Therefore, we include a substantial review on service science and service dominant logic in this section.

\subsection{Service Research Vocabulary}

Generally, the core vocabulary in modern service research is provided by ServiceDominant logic [4]. As shown in Figure 2, in its guidance to shift from a goods-centered view to a service-centered view, S-D logic proposes eight commensurate shifts in thinking: (1) a shift to a focus on the process of serving rather than the creation of goods, (2) a shift to the primacy of intangibles rather than tangibles, (3) a shift to a focus on the creation and use of dynamic operant resources as opposed to the consumption and depletion of static operand resources, (4) a recognition of the strategic advantage of symmetric rather than asymmetric information, (5) a shift to conversation and dialog as opposed to propaganda, (6) an understanding that the firm can only make and follow through on value propositions rather than create and add value, (7) a shift in focus to relational rather than transactional exchange, and (8) a shift to an emphasis on financial performance for information feedback rather than a goal of profit maximization.

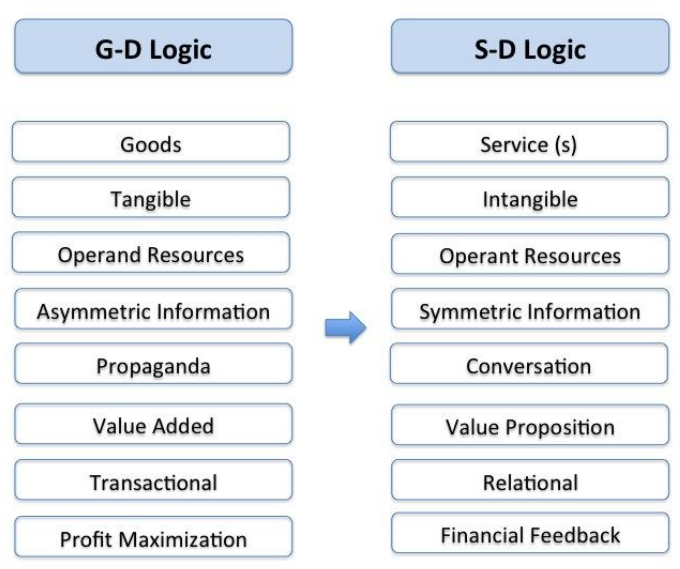

Figure 2. The prosed eight fundamental shifts in S-D logic

When a firm sees itself as a manufacturer with an implied purpose of selling what it makes, it sees the key to making more money as selling more and more goods. In contrast, the S-D logic suggests that since these manufactured goods are actually mechanisms for service provision, the customer is always buying a service flow rather than a tangible thing, and thus the firm should perhaps reconsider the nature of its offering [4]. S-D logic emphasizes importance of dynamic operant resources over static operand resources. The operand resources, such as natural resources, require something be done to it to be useful, where as 
dynamic operant resources are largely intangible and can produce an effect. The main operant resource it recognizes is knowledge, which is the only sustainable source of national wealth and competitive advantage. S-D logic also emphasizes the importance of exchanging symmetric information between the two parties and also the importance of having a conversation between the two parties than one party leading propaganda through advertising. The traditional G-D logic views value as a property of a good, which was added during the manufacturing process. Thus, the cost of adding value reflects the price the customer has to pay to acquire the good. However, S-D logic recognizes that value is not created and added at the factory but rather co-created by the customers' assessment of value-in-use. This involvement of customer implies that the firms can only make an offer of value creation through the application of its resources to some need of the customer- that is through a service. In other words, firms can only make a value proposition and if customers accept it, value will be co-created. Traditional G-D logic encourages maximizing profits by completing as much transactions as possible. In contrast, S-D logic advises to maintain longer relationships with customers using financial feedbacks as sources of information for learning to continuously generate and test new hypothesis that serve customers better.

According to [6], a society is a complex system of servicing actors. It could also be viewed as a value constellation that comprise of multiple service systems [18]. Service system has been defined as a dynamic value co-creation configuration of resources, including people, organizations, shared information (language, laws, measures, methods), and technology, all connected internally and externally to other service systems by value propositions [7]. In this definition, anything such as people, businesses, government agencies, etc., could be a service system. A similar view on servicing actors of a society could be found in [19], where it defines business and social organizations as viable systems. A dynamic and complex system of interacting service systems such as a society is called a service ecosystem [6].

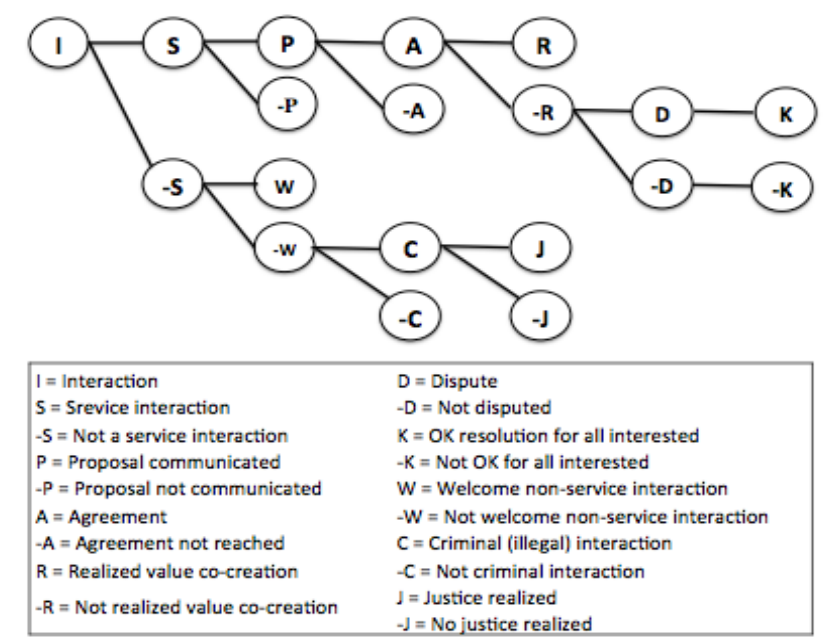

Figure 3. The ISPAR model of service system interactions

\subsection{ISPAR Model of Service System Interactions}

An interaction between two service systems has been presented as a model of ten possible outcomes in the ISPAR (Interact-Serve-Propose-Agree-Realize) model, which is illustrated in Figure 3 [7]. Each interaction between two service systems, according to the ISPAR model, could be either a service interaction or a non-service interaction. A service interactions leads to value co-creation where as a non-service interaction does not leads to value co-creation but 
rather has the potential for influencing value co-creation in future service interactions. For example, exchanging pleasantries with a future customer may affect the potential of value cocreation in the future service interactions.

According to the ISPAR model, a service interaction starts by communicating a proposal (P) by a customer to a service provider. Unless a proposal is communicated properly and understood by the other service system, a service interaction may not proceed. After the proposal communication the two service systems should come to an agreement for the service. Success of an agreement depends on some properties of the value propositions of the service systems or the fulfillment of certain requirements. For example, to get some services, the customer would need to possess proper identification documents. Similarly, to offer some services, the service providers have to possess a certain education level or a valid license. After coming to an agreement, the proposal will be realized and the service will be consumed. However, depending on the characteristics of the value propositions of the two service systems, value may $(\mathrm{R})$ or may not $(-\mathrm{R})$ be co-created. Being unable to co-create value sometimes may lead to a dispute (D), to which a satisfactory resolution may (K) or may not (K) be found.

A non-service interaction (-S) could be either welcome interaction (W) or a non-welcome interaction (-W). A welcome interaction would make a positive impact on future service interactions where as a non-welcome interaction would make a negative impact. A nonwelcome interaction could perhaps be a crime (C), to which justice may (J) or may not (-J) be realized.

\subsection{Kauffman's NKCS Model}

According to [6], even the tangible resources in the ecosystem we live in can be viewed in terms of service provision. For example, natural pollination of crops by insects and trees that help prevent erosion and protect the watershed are examples of service provision. Notably, with these service provisions entities of such ecosystems coevolve by adapting to each other's changes. For example, human consumption of timber affects the growth of trees, which in turn makes an impact on the watershed, affecting humans. Therefore, in [20] we proposed Kauffman's NKCS model, which was originally proposed to study the coevolution of biological species [15], as a modeling framework for service ecosystems.

The NKCS model defines a system of $S$ entities, each having $N$ attributes. Each entity is connected with $X$ other interacting entities in the system $(X<=S)$. Each attribute can be at $D$ number of states and a state value combination of all $\mathrm{N}$ attributes determines a state of the entity. Hence, each entity has $\mathrm{D}^{\mathrm{N}}$ possible states. In other words, a state could be considered as a point in an $\mathrm{N}$-dimensional hypercube, which has a total of $\mathrm{D}^{\mathrm{N}}$ points. Each point $d=\left\{d_{l}\right.$, $\left.d_{2 \ldots} d_{N}\right\}$ is associated with a utility value $f$ denoted by equation 1 .

$$
f(d)=\frac{1}{N} \sum_{i=1}^{N} f_{i}\left\{d_{i},\left[d_{i_{1}}, \ldots, d_{i_{K}}\right],\left[\left(d_{i_{11}}, \ldots, d_{i_{1 C}}\right), \ldots,\left(d_{i_{X 1}}, \ldots, d_{i_{X C}}\right)\right]\right\}
$$

Here, $f_{i}$

is the utility contribution of the $i^{\text {th }}$ dimension (attribute) and the total utility of a given point (state) is the average of the utility contributions of all $\mathrm{N}$ dimensions. A notable feature in the NKCS model is that the utility contribution of an individual dimension depends on: (1) its state, (2) K other dimensions of the same entity, (3) and $\mathrm{C}$ other dimensions of each of the $\mathrm{X}$ other connected entities in the system. For example, the system in Figure 4 has two entities $(S=2)$, each of which having seven attributes $(N=7)$. The two attributes are connected $(X=$ 1) and each attribute depends on two other attributes of the same entity $(K=2)$ and three other attributes of the other entity $(\mathrm{C}=3)$. Furthermore, according to Equation 1, the utility 
contribution of $2^{\text {nd }}$ attribute of Entity 1 depends on attribute 4 and 6 of the same entity and attributes 1, 4 and 6 of the opposite entity. These positions are picked randomly. This system is scalable due to the parameter $\mathrm{S}$ since more and more entities could be added to the system and the complexity could be controlled using parameters $\mathrm{N}, \mathrm{K}, \mathrm{C}$ and $\mathrm{X}$.

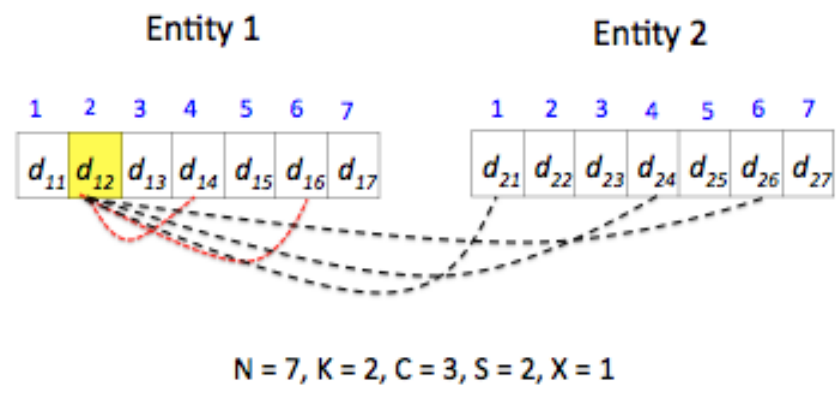

Figure 4. Dependency structure of two service system entities according to the NKCS architecture

The utility contribution of each dimension $f_{i}$ is determined by a function, which is defined by Equation 2 . Here, $R$ is drawn from a uniform distribution in the range $(0,1)$ to each of the $\mathrm{D}^{(\mathrm{K}+\mathrm{CX}+1)}$ inputs.

$$
f_{i}:\{0, \ldots, D-1\}^{K+X C+1} \rightarrow R
$$

These DN states and their respective utility values result in a rugged utility landscape of each entity in the system. In the original NKCS model, each entity has a representative agent, whose task is to continuously seek in the respective landscape for higher utilities. Each agent gets its turn to move from the current state to a new state in its one-mutant neighboring states [15], which give them a higher utility. However, due to the interdependency imposed by parameters $\mathrm{K}$ and $\mathrm{C}$, a move of an agent representing one entity to a new state alters the current utilities of other interacting entities. This induces those interacting species to look for better states in their respective landscapes at their respective turns to move. These moves by all entities result in coevolution in terms of utility. The model stops execution when all entities are better off at their current states. Further details on the NKCS model can be found in [15].

\subsection{Life cycle concept}

Life cycle concept is well known and widely used in management science. It distinguishes different stages of the life of a particular 'thing' in terms of a quantitative measure. For example, the Product Life Cycle (PLC) represents the unit sales curve for some product, extending from the time it is first placed on the market until it is removed [9]. The PLC, as shown in Figure 5, is approximated to a bell-shaped curve and usually divided into four stages, introduction/birth, growth, maturity and decline [21]. Even though the PLC is schematically approximated to a bell-shaped curve, as early as in 1979, [9] reports 12 identified patterns of the PLC including the bell-shaped pattern. Similarly, according to [10], the tourist destinations also follow a similar life cycle pattern, which they call the Tourism Area Life Cycle (TALC). They introduce an S-shaped curve with five distinguishable stages of evolution namely exploration, involvement, development, consolidation and stagnation. 
After stagnation stage, they propose that the curve would take one of five possible paths between rejuvenation and decline. Despite many criticisms as well as praises, the TALC concept is still being researched and cited even after three decades since it was first introduced [22].

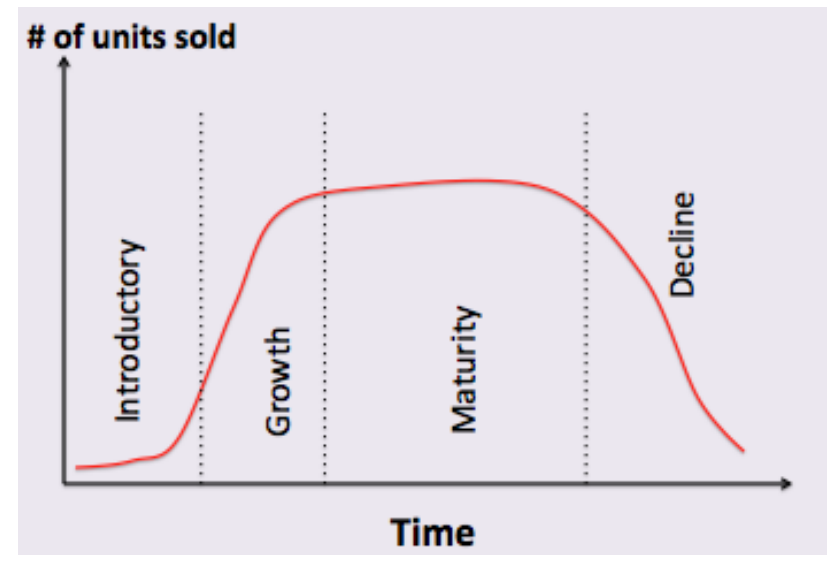

Figure 5. The basic product life cycle

\section{The agent-based model description}

The recent developments of agent-based modeling suggest using a common protocol for communicating agent-based models [16]. Therefore we describe our model adhering to the ODD protocol proposed by Grimm et al., [23]

\subsection{Overview}

3.1.1. Purpose: The purpose is to study the evolution of service systems with respect to a life cycle. In our hypothetical system comprising two types of service systems - customers and service providers - we specifically focus on the evolution of service providers.

3.1.2. State variables and scales: Our hypothetical service ecosystem has only two types of service system entities, i.e. service providers and consumers. A service system is represented as an 'agent' in the system and the number of agents of each type set at the initialization of the model. All service providers offer one particular service; for example, they all are beach hotels at a tropical island. Or else, they could be considered as fast food outlets near a university. According to [7], service systems connect to other service systems by value propositions. In other words, both customers and service providers should have a value proposition. Hence in our model, we define a distinct value proposition for the service provider entity and another distinct value proposition for the customer entity. According to [24], a value proposition can be defined by a set of value creating attributes. For example, in the value proposition of a hotel, connectivity (to the Internet), comfort, relaxation, etc. could be value-creating attributes. The hotel can mobilize its resources to these attributes to make an offer for value co-creation. For example, some hotels may provide Internet Hotspots or $\mathrm{Wi}$-Fi zones for the residents to connect to the Internet while some hotels provide in-room $\mathrm{Wi}$ Fi access.

We define a value proposition of $N$ attributes for each service system entity. Value proposition of service provider entity defines its service while the value proposition of customer entity helps defining customers. Furthermore, each attribute can be at two states, 0 
and 1. A state reflects a particular level reached by using resources, particularly knowledge. For example, some hotels may not offer its residents and Internet connection (state $=0$ ) and some will offer a Wi-Fi connection (state $=1$ ). Different agents of both service system entities could be at different states in their value propositions reflecting the reality. We define a state variable called current state (currentState) as an integer array for this purpose. For example, if $X$ and $Y$ are two agents of same type and $N=8, X$ could be at state 00001111 and $Y$ could be at state 10101010 . Current state of a service provider reflects its service level or service standard while the current state of a customer reflects his or her customer profile.

However, in reality, these value-creating attributes are not standalone. Their outcome depends on the states of many other known and unknown attributes. For example, the appeal of the hotel room will depend on the its design as well as other internal factors such as safety and maintenance and external factors such as the preferences of the customer that stay in. Therefore, we define each attribute of a given entity's value proposition to depend on K other attributes of the same entity and $\mathrm{C}$ other attributes of the other entity. This connectivity structure is similar to the system illustrated by Figure 4, even though $\mathrm{K}$ and $\mathrm{C}$ are two input parameters that could be initialized at the runtime. Using this structure, we manage to mimic an important, if not the most important, feature in service system interactions; the value cocreation. When a customer agent consumes the service from a particular service provider, the current state of the service provider is used to determine the value for that customer in that service. In other words, the final value realized by the customer (utility) is the average of the utility contributed by each attribute of the service provider's value proposition. However, due to the dependency structure imposed through parameters $\mathrm{K}$ and $\mathrm{C}$, the utility contribution by a given attribute of a service provider depends on its own state, states of $\mathrm{K}$ other attributes of the same entity and $\mathrm{C}$ attributes of the customer. In other words, for a customer to realize the full utility of a given attribute on service provider's value proposition, it is important to have the relevant attributes of his own value proposition at right states. For example, even if a hotel offers in-room Wi-Fi access, unless the customer possesses a laptop and knows how to connect to the Internet, he cannot realize the value of that offering. Similarly, the value of the service provider realized by a particular service interaction is determined by the average of the utility contributions of the attributes of customer's value proposition.

Initially, we set the current states of both customers and service providers randomly. It resulted in a pool of customers with diverse characteristics in their profiles as well as a diverse set of service providers at different service levels. In the current implementation, we set the current state of service providers to be constant, meaning that the service level of the service providers remains constant. However, the customers' current states change as they learn by experience and seek for better opportunities for value co-creation with different service providers.

Another important state variable is the 'expectation' both customer and service provider agents. Expectation of an agent is the adequate or minimum acceptable utility value from a service interaction. Expectation of a service provider is the minimum utility that makes him accept a service request by a customer. In our model, the expectation of each service provider is picked randomly within a range of upper and lower limits. Furthermore, the expectations of service providers are assumed to be constant throughout the simulation together with their service level (current state). However, the customers' expectation is considered as growing with time and experience. According to [25], there exists a zone of tolerance in customer expectation when they buy a service. The upper bound of the zone of tolerance is the desired service level, where as the lower bound is the adequate service level. We set the desired service level as $100 \%$ utility for all agents. However, the adequate service level of each customer agent is 
picked randomly within a specified range. According to [25], the adequate service level of a given customer grows with time when the customer gets experience. It means a once delightful service would become less attractive when experiencing the service over and over. Therefore as shown by Figure 6, we increase the adequate service level of a particular customer by a certain percentage determined by a variable called 'growth coefficient' after each successful value co-creation interaction.

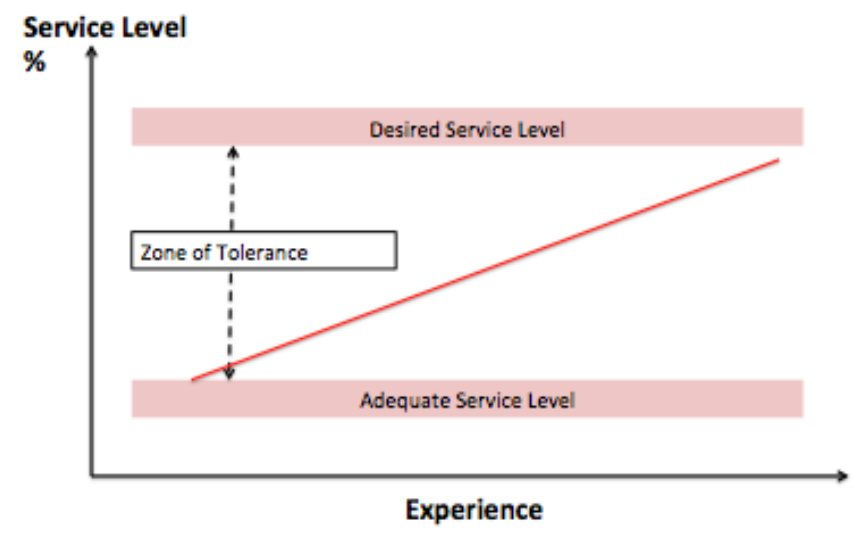

Figure 6. The change of customer expectation with time

Customers also have a variable that holds a memory about each service provider (memoryOfProviders) that he has previously interacted with. The memory contains the current states of the previously interacted providers. This memory is used when agents seek for a suitable service provider once he got the need. The frequency that the customer agents get the service need is controlled by a probability value (needProbability). If this probability is high, the customers get the service need very often. For example, fast food outlets serve for such a high frequent service need. Hotels basically serve for a low frequent need as people usually travel few times a year.

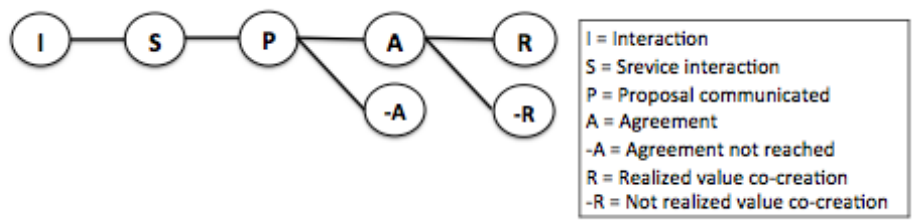

Figure 7. The branch of ISPAR model implemented in the model

3.1.2. Process overview and scheduling: The model basically considers one major process, i.e., the process of fulfilling a need of a consumer by a service. This process involves individual decisions of both customer and service provider agents. We use the ISPAR model of service system interactions explained before, as a guidance to implement this process. However, to make things simple, we use only a branch of the ISPAR model for the current implementation, which is shown in Figure 7. Here we assume that an interaction is always a service interaction and the agents are capable of communicating the service proposal effectively. Thus the major decision the service agents have to take is whether to agree on a service interaction or not. 
The process starts with a customer agent getting a service need. Once a customer agent gets a service need, the first task of the agent is finding a suitable provider. The agent first looks for a provider in the memory of providers and if it is empty (customer is a first-timer), a service provider will be picked randomly. If the memory is not empty, the customer agent evaluates the potential co-created value with each service provider and selects the one that is likely to give the highest value within his zone of tolerance. If a suitable provider could not be found yet, the customer agent seeks for previously unvisited providers and selects one randomly. Here we assume that the customer agents have a prior knowledge about all available service providers. If no provider could be found yet, the customer agent tries to change its current state to find a suitable provider. Following a greedy strategy [15], it evaluates all its one-mutant neighboring states for all service providers and selects the best provider and the corresponding new state. Moving to a new state by a customer agent reflects a change in the customer profile of that agent done by learning and adapting to the environment. Failing to find a suitable provider after all these attempts will lead the customer agent to give up the need and abort the service interaction.

Once a suitable provider is found, the consumer agent initiates an interaction by contacting the selected provider through a service request. This is similar to making a proposal in the ISPAR model. Here we assume that the proposal gets communicated without any misunderstanding. When a consumer agent makes a proposal, he transfers his current state to the service provider. The service provider checks the current state of the consumer to determine the potential value to him in the service interaction. For example, making a hotel room reservation will require a credit card number as a guarantee and if a customer fails to provide a valid credit card number, the hotel may reject the reservation request since the potential value is less than the expectation. If the potential value is higher than his expectation, the provider agent agrees to provide the service or reject otherwise. With the response of the service provider agent, the customer agent gets to know the current state of the service provider. Once the consent of the service provider is received (Agreement) for a service proposal, the service interaction comes to the value co-creation phase (Realize).

At the value co-creation phase, the consumer will determine the value of the service by evaluating the utility in the landscape. Since the total utility perceived by the customer from the service provider's value proposition depends on the current states of both the service provider and customer, value can be considered as co-created. If the perceived value is within the zone of tolerance of the customer agent, it becomes a realization of value co-creation $(\mathrm{R})$ as both parties get benefited from the interaction. Otherwise, it is not a realization of value co-creation (-R). Further details on the process overview and the ISPAR model can be found in [26].

\subsection{Design Concepts}

3.2.1. Emergence: We focus on the emerging pattern based on the number of service interactions that occur with each service provider at each time step. All service interactions that passed through the agreement phase (A) of the ISPAR model are counted for this purpose.

3.2.2. Adaptation: Only customer agents display an adaptive behavior. When they are unable to find a service provider who can meet their expectation, they search for a better state in their one-mutant neighboring states, where it is more likely that they can co-create value with one of the providers. Moving to the one-mutant neighboring states can be likened to the process of 
changing one's own customer profile along a given attribute. For example, a customer who didn't possess a credit card before and found it difficult to reserve a hotel room will try to buy a credit card before trying to reserve a hotel next time. This could be considered as a change in state.

3.2.3. Fitness/Objectives: In this implementation, only the customer agents have an objective. They try to meet their increasing expectations through successfully realized value co-creation interactions with available providers.

3.2.4. Prediction: If a customer agent interacted with a service provider before, his memory contains the current state (service level) of the provider, which helps to predict the potential for realized value co-creation in future interactions.

3.2.5. Stochasticity: If a customer agent consumes the service for the first time or if the customer agent finds that the service providers in his memory have no potential for further value co-creation, he selects a new provider randomly from the previously unvisited list.

\section{Results}

This section contains the results of the simulation based on the above model. For all our simulation runs, we set $\mathrm{N}=8, \mathrm{~K}=2$ and $\mathrm{C}=2$. The reason for selecting those values was none other than preserving computational resources to ensure faster execution of the simulation. We further set the number of consumer agents to 1000 and the number of provider agents to 5 . In our first experiment, we set the need probability to 0.25 to ensure a lower frequency of getting the service need. The result of the simulation is shown in Figure 8 . The five lines on the graph correspond to five random service providers offering the same value proposition but with different service levels. In other words, the initial states of the value propositions of those providers are different from each other. Moreover, those initial states are set to be constant throughout the simulation to reflect a fixed service level. In other words, the service provider agents in this simulation do not change their service level due to learning. The Y-axis corresponds to the number of service interactions done by each provider at a given time step.

In the second experiment, we setup the need probability to 0.95 to secure a high frequency in getting the service need. For example, the fast food industry serves for a high frequent need. The corresponding result is shown in Figure 9. Similar to the previous case, the five lines on the graph correspond to five service providers offering the same value proposition but at different levels of service. Notably, the value proposition in this case is not necessarily to be the same as in the previous case. In other words, the two simulation runs are mutually independent. Similar to the previous case, the Y-axis corresponds to the number of service interactions occurred between each service provider agent and customer agents.

\section{Discussion}

At this point, what we are interested in is the basic shapes of the curves on each graph. Looking at the two graphs, it is apparent that all the curves more or less display the properties of the known life cycle patterns. For example, in the graph in Figure 8, Provider01 goes through the typical bell-shaped life cycle pattern where as Provider02 and Provider03 follows almost a similar pattern. The other two providers follow patterns of unsuccessful businesses with less growth and subsequent decline. Similarly, the Provider01 and Provider03 on the graph in Figure 9 follow the typical bell-shaped life cycle pattern where as Provider02, 
Provider04 and Provider05 only go through some phases of the typical curve such as stagnation and decline. Hence, irrespective of the frequency of getting the need, we can come to a general conclusion that service systems go through a life cycle pattern with generally identifiable phases in the process of evolution. We propose this to be called the Service Life Cycle (SLC)

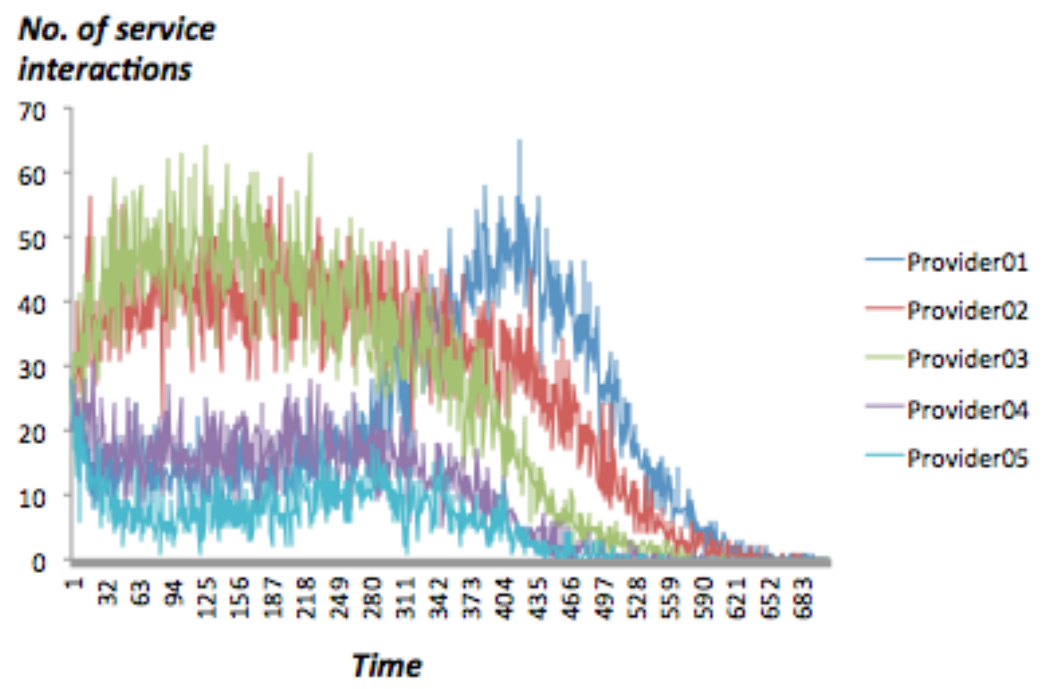

Figure 8. Results of four simulation runs with "Need Probability" $=0.25$

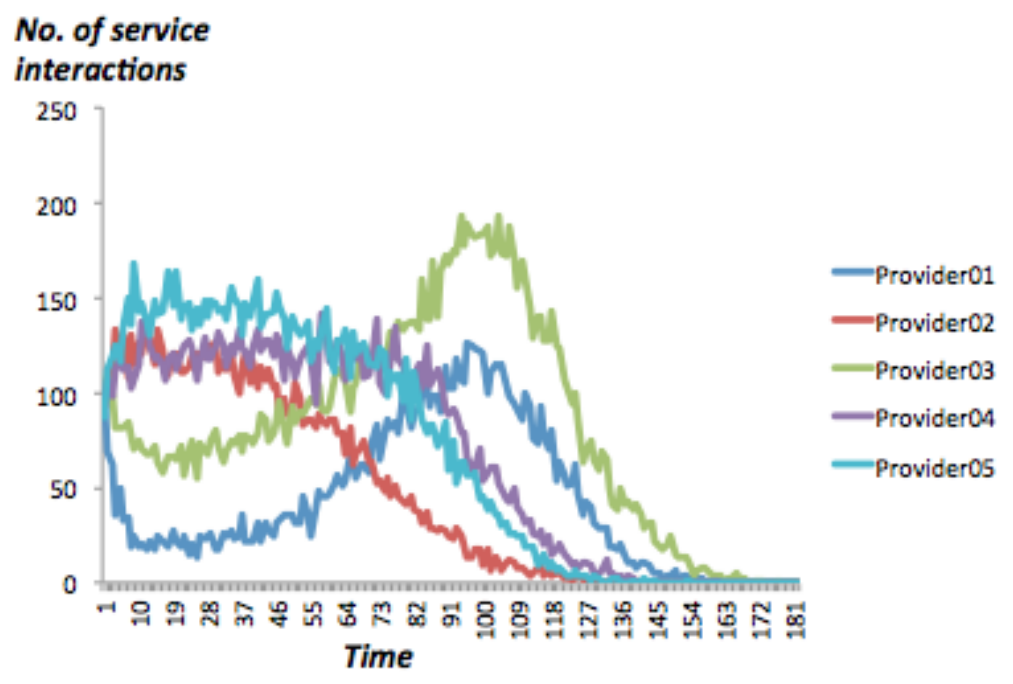

Figure 9. Results of four simulation runs with "Need Probability" = 0.95

Even though this result is preliminary, it makes a positive impact for the development of a unified theory of services based on S-D logic. The recognition of service system as the basic abstraction of service science gives a fundamental unit of analysis in understanding service ecosystems. The proposed service life cycle contributes to this endeavor by identifying an evolutionary path for service providers in terms of their offering. The proposed evolutionary 
path could be used as a reference to identify the performance of a given service system and investigate probable strategies for sustainability. Moreover, the service life cycle is capable of bringing in different life cycles discussed in the conventional management literature under a common umbrella. For example, both product life cycle and tourism area life cycle are service life cycles. A firm offering a product is a service system and so as a tourist destination. Thus, a common discussion with reference to the service life cycle would be beneficial for both manufacturing firms and tourist destination managers.

However, this model is incomplete in terms of S-D logic. The main drawback here is that the service providers' service level is kept constant. In fact, it was done so to help revealing the evolutionary pattern in the extreme case. However in practice, firm generally learn from environmental feedbacks and change through innovation. According to S-D logic, rather than trying to maximize profit by running excessive advertising campaigns, firms need to rely on conversation with customers and financial feedback to learn better ways to help customers cocreate value. Another drawback of the model in representing the reality is the lack of information exchange among customer agents. In reality, a purchase decision would be highly influenced by the information received from friends, relatives, neighbors, and social media. Generally, this falls into the category of non-service interactions of the ISPAR model. Therefore, our future work involves improving this model a true dynamic market model, which could be used to support service providers making decisions.

\section{Conclusion}

We intend to study the evolution of service systems to understand the dynamics of service ecosystems through Agent-based modeling approach. For this purpose we develop an agentbased model of an artificial market with two service system entities - service providers and customers. In the model, customers attempt to satisfy their need for a particular service by cocreating value with the offering of different providers in the system. Our analysis specifically focuses on the evolution of service providers in this context. The model is based on the guidance provided by service-dominant logic and we use Kauffman's NKCS architecture to model the two service system entities. Moreover, we use the ISPAR model of service system interactions to model the interactions between customer service systems (customer agents) and service provider service systems (service provider agents). This paper presents the details of the formulation of the model and a discussion based on the preliminary results. The results reveal that the evolution of service providers in the system follows a life cycle pattern. Therefore, we propose a unified service life cycle (SLC) as a reference to evaluate the performance of service providers in a system and test different strategies for sustainability.

\section{Acknowledgements}

We thank the Ministry of Education, Culture, Sports, Science and Technology of Japan for their support for our research through a scholarship

\section{References}

[1] A. L Ostrom, M. J. Bitner, S. W. Brown, K. A. Burkhard, M. Goul, V. S. Daniels, H. Demirkan and E. Rabinovich, "Moving forward and making a difference: Research priorities for the science of service", Journal of Service Research, vol. 13, no. 4, (2010), pp. 4-36.

[2] J. Spohrer and P. P. Maglio, "The emergence of service science: Toward systemic service innovations to accelerate co-creation of value", Production and Operations Management, vol. 17, no. 3, (2008), pp. 238-246.

[3] CIA, "The World Fact Book", https://www.cia.gov/library/publications/the-world-factbook/fields/2012.html, (2012). 
[4] R. F. Lusch, S. L. Vargo and A. J. Malter, "Taking a leadership role in global marketing management", Organizational Dynamics, vol. 35, no. 3, (2006), pp. 264-278.

[5] IfM and IBM, "Succeeding through service innovation: A service perspective for education, research, business and government", Cambridge, United Kingdom: University of Cambridge Institute for Manufacturing, (2008).

[6] S. L. Vargo and R. F. Lusch, "It's all B2B....and beyond: Toward a systems perspective of market", Industrial Marketing Management, vol. 40, no. 2, (2011), pp. 181-187.

[7] P. P. Maglio, S. L. Vargo, N. Caswell and J. Spohrer, "Service system is the basic abstraction of service science", Information Systems and e-Business Management, vol. 7, no. 4, (2009), pp. 395-406.

[8] P. P. Maglio and J. Spohrer, "Fundamentals of service science", Journal of the Academy of Marketing Science, vol. 36, no. 1, (2008), pp. 18-20.

[9] D. R. Rink and J. E. Swan, "Product life cycle research: A literature review", Journal of business Research, vol. 7, no. 3, (1979), pp. 219-242.

[10] R. W. Butler, "The concept of a tourist area cycle of evolution: implications for management of resources", The Canadian Geographer/Le Géographe canadien, vol. 24, no. 1, (1980), pp. 5-12.

[11] I. Ng, R. Maull and L. Smith, "Embedding the new discipline of service science", The Science of Service Systems, Springer US, (2011), pp. 13-35.

[12] T. Terano, "Beyond the KISS principal of agent-based social simulation", Journal of Socio-informatics, vol. 1, no. 1, (2008), pp. 175-187.

[13] J. M. Epstein, “Agent-based computational models and generative social science, Generative Social Science: Studies in Agent-based Computational Modeling”, Joshua M. Epstein (Eds.), Princeton University Press, Princeton, New Jersey, (2006).

[14] M. A. Janssen, "Complexity and eco-systems management: The theory and practice of multi-agent systems", Edward Elgar Publishing, Inc., Cheltenham, UK, (2000).

[15] J. Padget, R. Vidgen, J. Mitchell, A. Marshall and R. Mellor, "Sendero: an extended, agent-based implementation of Kauffman's NKCS model”, Journal of Artificial Societies and Social Simulation, vol. 12, no. 4, (2009), pp. 824-844.

[16] C. Rajapakse and T. Terano, "Analyzing the Evolution of Service Ecosystems with respect to Life Cycle Concept through Agent-based Modeling Approach", Proceedings of the $2^{\text {nd }}$ International Workshop on Smart Technologies for Energy, Information and Communication, Republic of Korea, (2013).

[17] R. Matteo, R. Leombruni, N. Saam and M. Sonnessa, "A common protocol for agent-based social simulation", Journal of artificial societies and social simulation, vol. 9, (2006).

[18] C. A. KielisZewski, P. P. Maglio and M. Cefkin, "On modeling value constellations to understand complex service system interactions”, European Management Journal, vol. 30, no. 5, (2012), pp. 438-450.

[19] R. Badinelli, S. Barile, I. Ng, F. Polese, M. Saviano and P. D. Nauta, "Viable service systems and decision making in service management", Journal of Service Management, vol. 23, no. 4, (2012), pp. 498-526.

[20] C. Rajapakse and T. Terano, "Modeling value co-creation process in complex service systems using Kauffman's NKCS architecture", Advanced Methods and Technologies for Agent and Multi-Agent Systems, D. Barbucha, M. T. Le, R. J. Howlett, L. C. Jain (Eds.) Frontiers in Artificial Intelligence and Applications series, vol. 252, ios Press, (2013), pp. 71-80.

[21] G. S. Day, "The product life cycle: analysis and applications issues", The Journal of Marketing, (1981), pp. 60-67.

[22] R. W. Butler, "Tourism Area Life Cycle”, Contemporary Tourism Reviews, Chris Cooper (Eds.), Goodfellow Publishers Ltd., (2011).

[23] G. J. Polhill, D. Parker, D. Brown and V. Grimm, "Using the ODD protocol for describing three agent-based social simulation models of land-use change", Journal of Artificial Societies and Social Simulation, vol. 11, no. 2, (2008).

[24] I. Ng, G. Parry, L. Smith, R. Maull and G. Briscoe, "Transitioning from a Goods-Dominant to a ServiceDominant Logic: Visualizing the Value Propositions of Rolls-Royce", Journal of Service Management, vol. 23, no. 3, (2012), pp. 416-439.

[25] V. A. Zeithamal and L. B. Leonard, "The nature and determinants of customer expectations of service", Journal of the Academy of Marketing Science, vol. 21, no. 1, (1993), pp. 1-12.

[26] C. Rajapakse and T. Terano, "An agent-based implementation of service system interactions based on the ISPAR model", In proceedings of the $8^{\text {th }}$ International Workshop on Agent-based Approaches in Economic and Social Complex Systems, Tokyo, Japan, (2013). 


\begin{abstract}
Authors

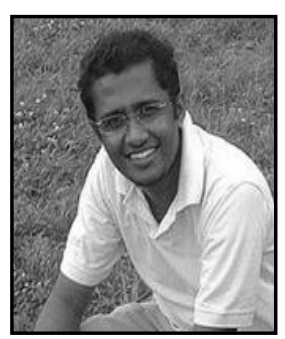

\section{Chathura Rajapakse}

Chathura Rajapakse is a second year doctoral degree candidate at the Terano laboratory of the Department of Computational Intelligence and Systems Science, Tokyo Institute of Technology, Japan. He possesses a B.Sc. degree in Industrial Management from the Department of Industrial Management, Faculty of Science, University of Kelaniya, Sri Lanka and a Master of Engineering degree from the Department of Computational Intelligence and Systems Science, Tokyo Institute of Technology, Japan. $\mathrm{He}$ is a recipient of the Japanese Government Scholarship and his current research interests include Service Science, Agent-based Modeling and Knowledge Management. He is also attached to the Department of Industrial Management, Faculty of Science, University of Kelaniya, Sri Lanka as a lecturer since 2007.
\end{abstract}

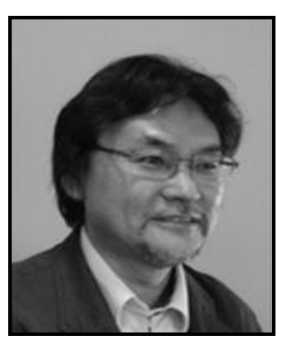

\title{
Takao Terano
}

Takao Terano is a professor at Department of Computational Intelligence and System Science, Interdisciplinary Graduate School of Science and Engineering, Tokyo Institute of Technology. He received BA degree in Mathematical Engineering in 1976 from University of Tokyo, M. A. degree in Information Engineering in 1978 from University of Tokyo, and Doctor of Engineering Degree in 1991 from Tokyo Institute of Technology. His research interests include Agent-based Modeling, Knowledge Systems, Evolutionary Computation, and Service Science. $\mathrm{He}$ is a member of the editorial board of major Artificial Intelligence and System science- related academic societies in Japan and a member of IEEE, AAAI, and ACM. He is also the president of PAAA. 